\title{
FOODBORNE ILLNESS AMONG SCHOOL CHILDREN IN GA EAST, ACCRA
}

\author{
K.L. MALM, ${ }^{1}$ K.M NYARKO ${ }^{2}$ A.E. YAWSON ${ }^{3}$ B. GOGO ${ }^{4}{ }^{4}$ A. LAWSON ${ }^{5}$ and E. AFARI ${ }^{2}$ \\ ${ }^{1}$ Ghana Health Service - Malaria Control Programme, P.O. Box KB 493, Korle-bu , Accra- Ghana, ${ }^{2}$ Univer- \\ sity of Ghana, School of Public Health - Department of Epidemiology and Disease Control, Box LG 13 Legon \\ Accra - Ghana, ${ }^{3}$ University of Ghana Medical School - Department of Community Health, Accra- Ghana and \\ National AIDS/STI Control Program - Clinical Care, DTD AR-West/Dansoman , Accra, Ghana, ${ }^{4}$ Ghana \\ Health Service, Ledzokuku Municipal Hospital, Accra- Ghana, ${ }^{5}$ Ghana Health Service - Disease Surveillance \\ Department, Accra- Ghana
}

DOI: $h t t p: / / d x . d o i . o r g / 10.4314 / g m j . v 49 i 2.2$

Corresponding Author: Dr. Keziah Laurencia Malm

E-mail:kezmalm@yahoo.com

Conflict of Interest: None declared

\section{SUMMARY}

Background: A food borne illness was reported in GaEast district of Greater Accra Region among school children in May, 2007 after eating food provided at school. The objective of the investigation was to determine the source, mode of contamination and the causative agent.

Methods: A case-control study was conducted, cases were schoolchildren with abdominal symptoms and controls were children of the same sex and class without any symptom during the same period. The school children were selected by systematic sampling. Food handlers and the children were interviewed by a structured questionnaire. Food handlers were physically examined and their stools and blood examined. The kitchen for food preparation was inspected. Risks of food borne infection from the foods eaten were determined using attack rates .

Results: The minimum, peak and maximum incubation periods were 2, 11 and 61 hours respectively. The source was rice and groundnut soup (with the highest attack rate difference). Stool and blood samples of food handlers were not infective. Storage facility for food items was poor. No food samples were available for organism isolation. A protocol to prevent such outbreaks was nonexistent.

Conclusion: The short incubation period and symptoms presented suggest an infective origin. The storage of the meat may potentially have been the point of contamination. The study showed that the schoolchildren ate contaminated food although the investigation could not determine the causative agent. Protocols to prevent such outbreaks need to be developed for the schools.

Keywords: Food borne, illness, contaminated food, school children, Accra

\section{INTRODUCTION}

The Government of Ghana instituted the Ghana School Feeding Programme (GSFP) in 2005 to provide onesquare-meal a day for pupils in basic public schools in most deprived areas of the country. ${ }^{1,2,3}$ GSFP is an initiative by Ghana to achieve the United Nations Millennium Development Goals (MDGs) on hunger, poverty and primary education. ${ }^{4}$

On $29^{\text {th }}$ of May, 2007 it was reported in the newspapers that a number of school children were reporting at various health facilities at Madina in the Ga-East district with diarrheoa and vomiting. ${ }^{5,6}$ It was reported that it occurred after they had taken food prepared for them under the school feeding programme the previous day. Children from three schools were affected namely Seventh Day Adventist Primary, Queen of Peace and the Madina Estates Schools. They had all eaten food prepared by the same caterer. This caterer has been preparing food for the children since the school-feeding program started in these schools for about six months.

Usually most children in the primary classes of the schools eat food prepared under the school feeding programme, a few of the children however do not eat food from the programme but rather eat food from the school canteens. 1,3

In this particular instance the food prepared by the programme was groundnut soup + riceballs + meat. It was not the first time that rice balls and groundnut soup had been prepared for the children. ${ }^{5}$ Foods served in the school canteens included 'waakye', 'kenkey' and 'banku'. The objective of this investigation was to characterize the people affected, determine source of infection, the mode of contamination and transmission and the causative agent. 


\section{METHODS}

A team was set up under the auspices of the Surveillance Department of the Ghana Health Service to investigate the outbreak. A case-control study was conducted in the three schools involved in the outbreak in Madina (a large community in the Eastern part of Accra) namely the Queen of Peace, Madina Estates and the Seventh Day Adventist primary schools. A total of 212 schoolchildren made of 106 cases and 106 controls were interviewed by a structured questionnaire. Children interviewed were in the primary school from kindergarten to class six (those eligible for feeding under the GSFP).

The number of cases selected was 49 from Madina Estate School, 38 from Seventh Day Adventist School and 19 from Queen of Peace School; selected number per school was weighted by the actual number of cases that occurred in that school.

Cases were children who had gastro-intestinal symptoms from the time the meal was eaten (i.e. $28^{\text {th }}$ of May, 2007) to a week after i.e. time of investigation. Cases were selected through a systematic sampling process.

Controls were children of the same sex and class as cases but who did not experience any gastro-intestinal symptoms during the outbreak period. Stool samples of some school children who reported at health facilities in Madina during the period of investigation were sent to the laboratory for examination.

Food handlers responsible for the provision of food for the schools were also interviewed. All food handlers were physically examined and their stool and blood samples were sent for laboratory examination. Inspection of the kitchen where food was prepared, how food was usually transported and how it was served was also undertaken. Risk of food borne infection from the various foods eaten by the school children was determined using attack rate difference.

The team investigated this outbreak under the auspices of Ghana Health Service and due to the fact that the Service has the legal mandate to ensure health and also because this was an acute occurrence, which would have outrun its course by the completion of the ethical approval process, no ethical approval was sought.

\section{RESULTS}

\section{Characterization of disease outbreak}

A total of 212 school children comprising 106 cases and 106 controls were administered questionnaires, as well as 7 food handlers. Even though the cases and controls were not matched on age they were very similar with respect to age. The mean age of the cases was 11.05 (range 6-16, std dev 2.14) and the mean age of the controls was 11.18 (range 4-18, std dev 2.63). Majority of the cases were in the $8-11$ year group, followed by the 12- 15 year group. Figure 1 which is the epicurve suggests a point source outbreak with minimum and maximum incubation period of 2 hours and 61 hours respectively. The peak incubation period was 11 hours as demonstrated in Figure 1.

\section{Figure1: Epicurve of Food Borne Illness Among Schoolchildren}

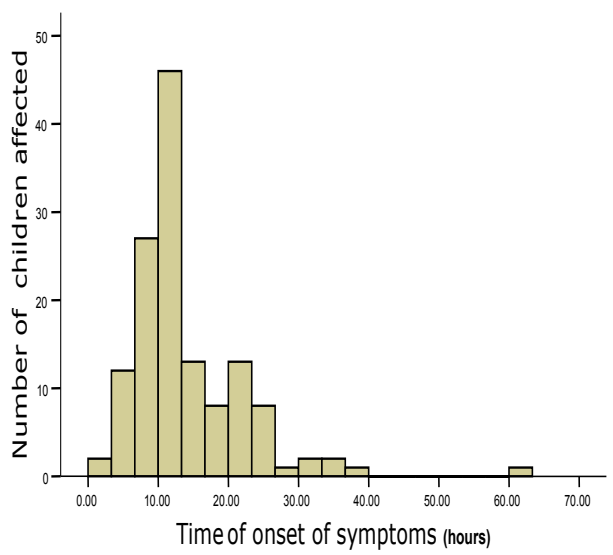

\section{Clinical spectrum of disease}

The most frequent symptoms suffered by the school children were diarrhoea and vomiting with 95 (90\%) children having multiple symptoms of diarrheoa, vomiting, abdominal pain and fever in varied combinations. The duration of illness ranged from 1 to 5 days with a mean duration of $2+/-0.9$ days.

Table 1 indicates that 104 (98\%) of cases and $69(65 \%)$ of controls ate the groundnut soup + rice ball + meat. The specific food attack rates as shown in Table 1 demonstrated that $98.1 \%$ attack rate for the groundnut soup + rice ball + meat meal.

Source of food borne illness: From Table 1, the groundnut soup + rice balls + meat meal (eaten together) prepared under the school feeding programme had the highest attack rate difference of 33 . 
Table 1 Specific food eaten by the School Children, Food Specific Attack Rates and Attack Rate Difference

\begin{tabular}{|c|c|c|c|c|c|c|c|}
\hline \multirow[t]{2}{*}{ Foods Served } & \multicolumn{2}{|c|}{ Cases (106) } & \multirow{2}{*}{$\begin{array}{l}\text { Attack Rate } \\
(\%)\end{array}$} & \multicolumn{2}{|c|}{ Controls (106) } & \multirow{2}{*}{$\begin{array}{l}\text { Attack Rate } \\
(\%)\end{array}$} & \multirow{2}{*}{$\begin{array}{ll}\text { Attack } & \text { Rate } \\
\text { Difference } & \end{array}$} \\
\hline & Ate & Did Not eat & & Ate & Did Not eat & & \\
\hline $\begin{array}{l}\text { Groundnut soup + rice ball } \\
+ \text { meat }\end{array}$ & 104 & 2 & 98.1 & 69 & 37 & 65.1 & 33 \\
\hline Waakye & 1 & 105 & 0.9 & 20 & 86 & 18.9 & -18 \\
\hline Banku and okro soup & 1 & 105 & 0.9 & 13 & 93 & 12.3 & 11.4 \\
\hline Kenkey and Fish & 0 & 106 & 0 & 4 & 102 & 3.8 & -3.8 \\
\hline Total & 106 & & & 106 & & & \\
\hline
\end{tabular}

\section{Laboratory Investigation: Causative agent:}

There was no available food sample at the time of investigation for analysis. Most of the school children that reported at the various health facilities did not have their stools taken for laboratory investigation before commencement of antibiotics. Only three of the children had their stools examined for the presence of pathogens and also cultured but this did not show any pathogens, because antibiotics had been started. Stool samples of all the food handlers were taken but there was no pathogen detected on laboratory examination.

\section{Inspection of Food preparation, storage, distribution and consumption}

Only two out of seven food handlers had valid health certificates required for food handlers. No obvious skin infection was noticed on the hands of the food handlers and none of them were carriers of any infective agents upon laboratory examination of their stools.

The ingredients for the food including the meat were bought from one of the markets in Accra, it was not possible to illicit how and where the meat was slaughtered and how it was transported after slaughtering. The kitchen where food was prepared was neat and all food handlers were appropriately dressed. The food was prepared through boiling on a locally made stove using charcoal. Food was transported in closed icechest covered with sheets in a pickup.

Those who served the food for the children in the schools washed their hands with water but never with soap before serving the food. The mode of serving was such that their hands were directly in contact with the food they served the children to eat.

All the classes had adequate number of plates and cutlery for the schoolchildren, which were usually washed after use each day and stored in their class cupboards. These bowls were however not washed again just before they were used.
There was also no water and soap for the school children to wash their hands before eating especially for the children who for any reason did not use cutlery.

\section{Mode of contamination}

The storage system at the kitchen for food items was not adequate. Due to the frequent power outages, the freezer was unable to maintain the right temperature to keep products frozen. There was water that had drained from meat/fish placed in the freezer which was a ready medium for growth of pathogens. The possible source of contamination might have been the meat probably due to poor storage and subsequently improper boiling in preparation of the soup. The fluid from the freezer could not be cultured due to some logistical challenges and non-availability of samples of the raw meat used for the cooking of the particular groundnut soup that the children ate.

\section{Other important observation}

There was no laid down protocol for emergencies being implemented by either the health workers or schools. Parents were at a loss what to do apart from just seeking medical care for their wards. We did not observe any laid down protocol under the school feeding programme in terms of ensuring that the food handlers had the requisite laboratory certificates; monitoring for any food borne diseases associated with the feeding programme or letting the schools or the general public know what to do in case of such outbreaks.

\section{DISCUSSION}

The fact that a number of school children came down with gastrointestinal symptoms mostly after eating the meal from school indicated that there was a food borne illness. The most likely source was the groundnut soup + rice balls ++ meat because of the comparatively higher attack rate difference associated with it. The mode of contamination might have been through the process of storage of the meat though this was not confirmed by any laboratory investigation of the fluid from the freezer due to logistical challenges and non-availability of samples of the raw meat used for the cooking. 
The food might not have been adequately cooked to kill whatever pathogens might have contaminated the food. The presence of fever as one of the common presenting symptoms and the short incubation period makes it likely to be of infective origin though this is not conclusive. The negative attack rate difference seen with the waakye and the kenkey and the fish might be due to the low number of children who ate those foods. Analysis of beef and goat meat in Accra by Newman in 2005 showed that they were contaminated with unacceptably high levels of bacteria.

Even a supermarket sample of meat (displayed under refrigeration) was found to have Shigella flexneri. ${ }^{7}$ Production and processing of meat in the city of Accra and other towns around the country leaves much to be desired. Although abattoirs are available in the city, a lot of butchers kill and process animals under unsanitary conditions. Transportation of meat is done in car boots and other unapproved vans to the market where it is then exposed to flies and dust. Open display of meat in the market for several hours exposes it to more flies, which are known vectors of bacteria including coliforms. $^{7,8}$

The investigating team arrived a few days after the children had eaten the food it was not possible for us to get samples of the food eaten for laboratory examination. Most of the school children who fell ill during the outbreak and visited the health facilities around did not have their stools examined. No specific causative agent was therefore isolated. These were major limitations identified by the investigating team; however the findings made are essential for developing policies to improve Ghana's School Feeding Programme., 3,9

The findings from the investigation indicated that there were no laid down emergency preparedness plan for the health workers neither was there a protocol for the school feeding programme in practice. In this particular incident there were no reported deaths, had the outbreak resulted in deaths so many children may potentially have died because of the generally poor emergency response system that characterize most basic schools in the country.

Studies on food borne outbreaks in schools and public institutions in developed countries have found such emergency response systems as very essential and efficient in containing the effects of food related disease outbreaks. $^{10,11,12}$ Public health education and assessment of food handlers on hygienic food storage and preparation in basic schools and other educational institutions (where food is prepared and served to many pupils and students) is an essential health policy that ought to be seriously considered.
It is more efficient to prevent these food borne illnesses among school pupils than managing the after effects. ${ }^{7,8}$

The Ghana School Feeding Programme is a good national initiative with enormous public health benefit to schoolchildren especially in the deprived areas of the country. Indications are that the children enjoy the food, and staff who provide the children with the food are willing to provide the best of services and therefore all the necessary measures should be put in place for its successful implementation and sustenance.

\section{RECOMMENDATIONS}

The survey made these recommendations: Routine monitoring and surveillance of food borne diseases by School Feeding Programme in collaboration with the Ghana Health Service represented at the district level by the District Health Management Team should be undertaken.

The provision of guidelines by the District Assembly in collaboration with the District Health Management Team as to what teachers and parents should do in case of outbreak should be developed and communicated to stakeholders. In addition reporting on food borne diseases by health facilities should be implemented (at least reporting should be biannually with a requirement for zero reporting).

The District Assembly should ensure the availability and implementation of protocol for food handlers to undertake required medical examinations expected of them at the right time. Finally, the Ministries of Health and Education need to provide the necessary guidelines and protocols for handling emergency food related disease outbreaks in public educational institutions.

\section{CONCLUSION}

The schoolchildren ate food under the school feeding programme, which had been contaminated. The source of the contamination and the causative agent could not be identified in this study, though the meat used for preparing the food was suspected to be the source because of the state of the storage of the meat.

There was however no laid down emergency preparedness plan for such outbreaks, neither was there a protocol to guide the school feeding programme in practice. The school feeding programme is a good programme contributing to improving the health and educational standards of children in the nation. Therefore increased efforts by all stakeholders are essential to ensure the success of the programme. 


\section{REFERENCES}

1. Ghana School Feeding Programme (GSFP) and Partnership for Child Development (2011), Planning for action. Published by GSFP, Ministry of Local Government and Rural Development, 2011, Accra, Ghana

2. Government of Ghana (GoG) Budget Statement and Economic Policy for 2010 Fiscal Year, page 197. Ministry of Finance and Economic Planning, Accra, Ghana, 2009

3. Ghanadistricts.com Report. A Public-Private Partnership between Ministry of Local Government and Rural Development and Maks Publications \& Media Services, 2006. Retrieved on 05-01-2013

4. Government of Ghana (GoG), Budget Statement and Economic Policy for 2006 Fiscal Year. Ministry of Finance and Economic Planning, Accra, Ghana, 2005.

5. The Daily Graphic, National Daily Newspaper, Accra, Ghana: May 29th, 2007.

6. http//www.myjoyonline.com/Ghana. Official webpage of a private broadcasting institution in Accra, Ghana Site visited and information retrieved on 04-01-13
7. Newman M.J. Take life easy; eat, drink and be merry. Editorial Commentary Food Safety Ghana Med J 2005:39 (2)

8. Mensah P, Armar-Klemesu M, Hammond AS, Haruna A, Nyaku R.Bacteria contaminants of lettuce, tomatoes, beef and goat meat from the Accra metropolis. Ghana Med J 2001; 162-167

9. http://www.modernghana.com. Feature Article on Ghana: School Feeding Program; more must be done. By Fidel Y. Tetteh, published on 31 January 2010. Retrieved on 05-01-2013

10. Public Health Agency of Canada Lessons Learned: Public Health Agency of Canada's Response to the 2008 Listeriosis Outbreak. Published by the Public Health Agency of Canada, December 2008.

11. Center for Disease Control and Prevention (CDC). Health and Safety: Foodborne Illness, Foodborne Disease, (sometimes called "Food Poisoning"). www. cdcinfo@cdc.gov. Information Retrieved on 02-01-2013

12. Charles Muruka, John Njuguna, Andrew Muruka, Kennedy Otuto, Margaret Maureen Atieno Oketch. Food Poisoning Investigation in an Underresourced Environment: A Case Report. Food and Public Health 2011; 1(1): 6-10 DOI: 10.5923/j.fph.20110101.02 